\title{
Wave Drag Reduction of SC(2)0410 Airfoil using New Developed Inviscid Compressible Adjoint Method
}

\author{
R. Kamali Moghadam ${ }^{1 \dagger}$, H. Jalali ${ }^{1}$ and A. Haghiri ${ }^{2}$ \\ ${ }^{1}$ Aerospace Research Institute, (Ministry of Science, Research and Technology), P. O. Box:14665-834, \\ Tehran, Iran \\ ${ }^{2}$ Imam Hossein University, Babaei Highway, Tehran, Iran \\ $\dagger$ Corresponding Author Email: rkamali@ari.ac.ir
}

(Received September 12, 2019; accepted December 29, 2019)

\begin{abstract}
A supercritical airfoil is geometrically optimized using the new developed adjoint compressible lattice Boltzmann method. Minimizing the drag coefficient and eliminating the shock wave on the supercritical airfoil surface are considered as the cost function with constraint of fixed lift coefficient. The continuous adjoint method is applied to able designers to implement large number of design variables in actual optimization problems. The adjoint equation based on the specified cost function and constrains is successfully derived. Discretization of the governing equations is carried out using the finite volume approach and $3^{\text {rd }}$ order of the MUSCL scheme. The supercritical SC(2)0410 airfoil, which has a strong shock on the top surface at transonic cruise conditions, is numerically optimized using the inviscid developed algorithm to eliminate the shock and reduce the wave drag. To validate the obtained results and show viscosity effect on the results, the base airfoil and optimized one are experimentally tested in a transonic wind tunnel at the same conditions. Pressure distribution on the surface of both the base and optimal airfoil are extracted from the experimental tests and compared with those of numerical simulations. The results indicate that the developed approach can be properly used for supercritical airfoil shape optimization for elimination the shock and reduction the wave drag.
\end{abstract}

Keywords: Adjoint approach; Aerodynamic shape optimization; Transonic wind tunnel; Supercritical airfoil; Wave drag reduction; Lattice Boltzmann method.

\section{INTRODUCTION}

Wave drag reduction in supercritical airfoils is always one of key challenges in design of transonic airplanes. Thus, airfoil shape optimization for eliminating the shock wave on the airfoil surface at such conditions to reduce the wave drag has been noticed by researchers for many years.

In many different optimization algorithms, the adjoint method has been well-known and applicable in aerodynamic problems. This algorithm can efficiently reduce the computation cost, since the computational expense incurred in the calculation of the complete gradient is effectively independent of the number of design variables (Jameson 1998). Jameson et al. (2003), (2005), actually could use CFD in shape optimization for different configuration such as airfoil, wing, wing-body and etc. They have successfully used the adjoint shape optimization with vast ranges of flow equations in macroscopic level; such as the Potential, Euler, NS, RANS and etc. equations. Pishevar et al. (2013) and Farrokhfal et al. (2014) applied the adjoint method with the Euler equation for optimization of the rotor blade and its plan form. Leoviriyakit (2005) derived the adjoint scheme based on the RANS equations and applied it on airfoil and wing plan form optimization. Anderson and Venkatakrishnan (1999) applied this approach based on the NS equations and unstructured grids.

Most of research in the adjoint method is performed based on the traditional CFD flow solvers like the Potential, Euler and NS equations. The Lattice Boltzmann method (LBM) has been developed as an alternative for the computational fluid dynamics approaches in simulation of fluid flows (Kadanoff et al. 1989 and Hardy et al. 1973). Specific features of the LBM against to the traditional CFD methods such as inherent parallelizability, simple explicit governing equation, easy access to solve pressure field, avoidance of nonlinear convective terms unlike the Navier-Stokes/Euler equations and so on, result in its vastly using, 
development and improvement in numerical simulations (Frisch et al.1987).

The standard LBM is extremely limited to simulate incompressible flows (Kadanoff et al. 1989) because it is constructed basis on Maxwellian expansion with low Mach number assumption. However, there are many numerical efforts for developing the LB models to simulate high Mach number compressible flows in recent years (Hardy et al. 1973, Frisch et. al. 1987, Alexander et. al. 1992, Kun et. al. 2001, Sun et. al. 1998). At the first, collision rules on the lattice Boltzmann equation (LBE) was constructed by Frisch et. al. (1987). Alexander et. al. (1992) implemented the LBM to analysis compressible flows and simulated the Berqures equation. Kun et. al. (2001) presented the BGK-models for viscous compressible flows by entering a non-equilibrium term to particle distribution functions. Sun et. al. (1998) developed a locally adaptive LB model by adding a mean flow velocity to local velocity of particles for simulating high speed flows. Recently, Hi et al. (2013) developed a 3-D LB model based on double-distribution function (DVBE) and solved it via the finite difference scheme. Qu et al. (2007) presented a new fantastic and simple method for evaluating distribution function that cover high speed flow regime. They used circular function $(\mathrm{CF})$ instead of the Maxwellian distribution function. They simulated many gas dynamic benchmark problems and also validated applied problems such as transonic flow over a Rae 2822 airfoil. The Qu method was very efficient and applicable for development of a new LB model and lattice in all flow regimes (Qu et al. 2005 and 2010). So, in this paper, the Qu method based on the CF idea was used and developed with some numerical efforts to capture discontinuities caused in compressible flow fields like contact discontinuities and shock waves. Very little research has been carried out in integration of the LBM and adjoint scheme. Major studies in this field have been restricted to topology optimization; however, this integration has been lightly used in aerodynamic shape optimizations. Pingen et al. (2009) used the standard LBM and adjoint algorithm for topology optimization and sensitivity analysis. Recently, Hekmat et al. (2015) developed the adjoint technique based on the standard LBM and applied it for flow optimization of the channel flow. Some researchers like Li et al. (2018) or Ngnotchouye et al. (2011) who have combined the adjoint approach and the LBM for the inverse problem, have developed it just for incompressible conditions using the Maxwell distribution function. There are several studies in developing the continuous-adjoint based optimization using the lattice Boltzmann method (Kreissl et al. 2011, Pingen et al. 2008, Tekitek et al. 2006 and Vergnault et al. 2014).

However, all of these developed methods have been restricted to use the standard LBM in incompressible flows or optimize the flow properties.

According to authors' literature review, the adjoint method based on the inviscid compressible LBM has not been developed for aerodynamic shape optimization. Derivation of the adjoint inviscid compressible LBM based the circular function to study the supercritical airfoil optimization problem has been performed by the authors for the first time. Due to universality of Boltzmann equation against the Euler/NS equations, the LBM is selected as flow solver due to its power and to be economical for simulation of large range of Knudsen number of flow (Chenghai and Hsu 2003). Also, the circular function is chosen as distribution function instead of the Maxwell distribution function due to its mathematical simplicity and flexibility of new lattice definition in simulation of compressible flowfield (Qu et al. 2007). Then, the adjoint method is selected as the optimization algorithm for calculation of the objective function gradient vector. Finally, the steepest decent technique is utilized as the gradient optimizer due to fast convergence to the optimize conditions. The finite volume method is also applied to discretize the governing equation which is suitable for optimization of both the compressible and incompressible inviscid flow. The developed procedure can be used for shape optimization at vast flow regimes.

Another innovation is to validate the developed algorithm by comparison of numerical shape optimization of the supercritical SC(2)0410 airfoil at cruise conditions with experimental test results in transonic wind tunnel. In this paper, airfoil optimization is performed with the objective function of minimizing the drag coefficient and eliminating the shock wave on the airfoil surface with constraint of fixed lift coefficient. The optimal airfoil profile is numerically extracted by developed inviscid algorithm; however, the obtained profile is experimentally tested in the transonic wind tunnel with viscosity effect. Pressure distribution on the surface of both the base and optimized airfoil are extracted from experimental tests and compared with those of numerical simulations. Therefore, effect of viscosity is investigated in eliminating the shock wave of optimized airfoil as the objective function. The results indicate that the developed approach can be properly used for airfoil shape optimization.

\section{GOVERINIG EQUATIONS}

In this section, the governing equations for the compressible LBM are explained and the new derived adjoint equation based on the flow governing equation is presented.

\subsection{Compressible Lattice Boltzmann Method}

The lattice Boltzmann method is developed based on the mesoscopic kinetic equations. Since the macroscopic fluid dynamics is result of the mesoscopic particles behavior, the Boltzmann equation defines the statistical concept of a system by expression of the density distribution function $f(x, t)$. General form of the Boltzmann equation with the BGK collision operation is expressed as $(\mathrm{Qu}$ et al. 2007):

$\frac{\partial f_{i, v}}{\partial t}+e_{i} \cdot \nabla f_{i, v}=\Omega_{i}\left(f_{i, v}\right)$ 
$\Omega_{i}\left(f_{i, v}\right)=\frac{1}{\tau}\left(f_{i, v}^{e q}-f_{i, v}\right)$

$i=1, \cdots, M$

$v=1, \cdots, L$

where, $f_{i, v}$ denotes the probability of existing a particle at time $t$ in location of $x$ with velocity of $e_{i}$ and $f_{i, v}^{e q}$ is equilibrium distribution function. In this research, the discrete velocities are defined in $M=$ 13 discrete velocities and $L=2$ energy levels which are presented for 2D directions on each lattice. As the LB grid is fixed, the problem should be expressed on grid scale.

The standard lattice Boltzmann equation is valid only for low-speed and incompressible flows (Chenghai and Hsu 2003). The basic Maxwell distribution function cannot be used in the standard LB models for simulation of compressible flows because it does not consider the rotational degree of freedom for diatomic or polyatomic molecules. In this paper, the circular function method is used to calculate the equilibrium distribution functions and remove limitations of the LBM in simulation of the compressible flows.

The circular function $\left(g_{c}\right)$ means that all mass, specific momentum and energy are concentrated on a circle located in a space of $\xi=(\xi, \eta, \lambda)$, where $(\xi, \eta)$ denotes the coordinate directions and $\lambda$ is the specific energy levels (Qu et al. 2007).

$\mathrm{g}_{c}= \begin{cases}\frac{\rho}{2 \pi c} & \text { if }\|\xi-\boldsymbol{u}\|=c \equiv \sqrt{D(\gamma-1) e} \\ 0, & \text { and } \lambda=\left[1-\frac{D}{2}(\gamma-1)\right] e \\ \text { otherwise. }\end{cases}$

$\boldsymbol{u}$ is the mean flow velocity, $c$ is effective peculiar velocity, and $\mathrm{D}=2$ is the spatial dimension. Although this function is very simple, it satisfies all needed statistical relations to make the BGK model recover the compressible the NS equations. The circular function is discretized onto some fixed points in the space of $\xi$ to construct the LB model. The Lagrangian interpolation is adopted to assign the circular function onto a set of discrete points without small Mach numbers assumption. In the space of $\boldsymbol{\xi}$, there are $M$ discrete points which the circular function is discretized onto all directions. The density for each discrete point, $i$, and velocity, $e_{i}$, is determined using a weight function $\varphi_{i, v}(\xi, \lambda)$ as:

$\rho_{i, v}=\frac{\rho}{2 \pi c} \oint \varphi_{i, v}(\xi, \lambda) d s$

The final circular equilibrium functions, $f_{i, v}^{e q}$, can be obtained using this weight function (Qu et al. 2007):

$f_{i, v}^{e q}=\frac{\rho}{2 \pi c} \oint \varphi_{i, v}(\xi, \lambda) d s$

\subsection{Definition of Optimization Problem}

The optimization problem is defined such that certain objective function should be minimized. In this paper, the objective function is to minimize the drag coefficient with constrain of the constant lift coefficient at cruise conditions. The design variables include all of surface points of airfoil geometry at y coordinate and the angle of attack. Therefore, the cost function gradient vector, $\delta \mathrm{I}$, is calculated versus flow properties and points position on the airfoil surfaces. Finally, the obtained gradient vector is changed by the steepest descent algorithm to arrive to the desired conditions at steady state.

\subsection{Continuous Adjoint Lattice Boltzmann Equation}

In this section, adjoint lattice Boltzmann equation is derived for the mentioned optimization problem. The main objective function, $I$, is defined as drag minimization at constant desired lift coefficient:

$I=\min C_{d}$

Design variables are angle of attack, $\alpha$ and airfoil surface points $\Gamma$. So, variation of the objective function due to design variables can be calculated as:

$\delta I=\delta C_{d}=\left.\frac{\partial C_{d}}{\partial \alpha}\right|_{\Gamma} \delta \alpha+\left.\frac{\partial C_{d}}{\partial \Gamma}\right|_{\alpha} \delta \Gamma$

According to aerodynamic constraint of design problem, another objective function should be considered to keep constant the lift coefficient as:

$I^{\prime}=C_{l}-C_{l_{d}}$

where, $C_{l_{d}}$ is the desired lift coefficient. Then the adjoint equation has to be solved for both the objective functions (Eq. 5 and 7) and the gradients are calculated in each design cycle.

Variation of the constant lift coefficient is zero, so variation of the angle of attack can be computed as:

$$
\begin{aligned}
& \delta C_{l}=\left.\frac{\partial C_{l}}{\partial \alpha}\right|_{\Gamma} \delta \alpha+\left.\frac{\partial C_{l}}{\partial \Gamma}\right|_{\alpha} \delta \Gamma=0 \\
& \delta \alpha=-\left(\left.\frac{\partial C_{l}}{\partial \Gamma}\right|_{\alpha} /\left.\frac{\partial C_{l}}{\partial \alpha}\right|_{\Gamma}\right) \delta \Gamma
\end{aligned}
$$

By substituting the variation of angle of attack in Eq. (8), variation of the main objective function due to design variables can be calculated as:

$$
\begin{aligned}
& \delta I=\left(\left.\Phi \frac{\partial C_{l}}{\partial \Gamma}\right|_{\alpha}+\left.\frac{\partial C_{d}}{\partial \Gamma}\right|_{\alpha}\right) \delta \Gamma \\
& \Phi=-\left.\frac{\partial C_{d}}{\partial \alpha}\right|_{\Gamma} /\left.\frac{\partial C_{l}}{\partial \alpha}\right|_{\Gamma}
\end{aligned}
$$

The drag and lift coefficients can be defined versus surface pressure coefficients $\left(C_{p}\right)$ as:

$$
\begin{aligned}
& C_{d}=-\oint_{c} C_{p}\left(S_{22} \sin \alpha-S_{21} \cos \alpha\right) \\
& C_{l}=-\oint_{c} C_{p}\left(S_{22} \cos \alpha+S_{21} \sin \alpha\right)
\end{aligned}
$$

where

$$
S_{21}=\left(\frac{d S}{d \xi}\right) d \xi, \quad S_{22}=\left(\frac{d S}{d \eta}\right) d \eta
$$

$S$ is the surface function and $(\xi, \eta)$ are the 
coordinate directions in computational plane. For computing the objective function variation, the Eq. (1) should be rewritten in the computational plane for steady state conditions:

$e_{i 1} \frac{\partial f_{\mathrm{i}, v}}{\partial \xi}+e_{i 2} \frac{\partial f_{\mathrm{i}, v}}{\partial \eta}=\Omega_{i}\left(f_{\mathrm{i}, v}\right)$,

where $e_{i 1}$ and $e_{i 2}$ present the contravariant velocities in $(\xi, \eta)$ directions, respectively. By substituting variation of the pressure coefficient in Eq. (9), and using the Lagrange multiplier method to the Eq. (14), then variation of the objective function can be calculated as:

$$
\begin{gathered}
\delta I=-\frac{1}{q_{\infty} \bar{c}} \oint_{c} \delta p\left[\Phi\left(S_{22} \cos \alpha+S_{21} \sin \alpha\right)-\right. \\
\left.\left(S_{22} \sin \alpha-S_{21} \cos \alpha\right)\right] d \xi \\
-\oint_{c} C_{p}\left[\Phi\left(\delta S_{22} \cos \alpha+\delta S_{21} \sin \alpha\right)+\right. \\
\left.\left(\delta S_{22} \sin \alpha-\delta S_{21} \cos \alpha\right)\right] d \xi \\
+\sum_{i=1}^{M} \sum_{\nu=1}^{L} \int_{D} \psi_{i, \nu} \delta\left[J e_{i 1} \frac{\partial f_{i, v}}{\partial \xi}+J e_{i 2} \frac{\partial f_{i, v}}{\partial \eta}-\right. \\
\left.J \Omega_{i, v}\left(f_{i, v}\right)\right] d \xi d \eta
\end{gathered}
$$

where $J$ is the metric Jacobian and $q_{\infty}$ is the free stream dynamic pressure. After some algebraic, and eliminating the gain of flow variation, the adjoint equation is derived as:

$$
\frac{\partial \psi_{i, v}}{\partial t}+e_{i 1} \frac{\partial \psi_{i, v}}{\partial \xi}+e_{i 2} \frac{\partial \psi_{i, v}}{\partial \eta}=\frac{1}{\tau}\left(\psi_{i, v}-\psi_{i, v}^{e q}\right)
$$

where $\psi_{i, v}$ is adjoint distribution function and $\psi_{i, v}^{e q}$ is adjoint equilibrium distribution function which is defined as:

$$
\psi_{i, v}^{e q}=\sum_{j=1}^{M} \sum_{v=1}^{L} \psi_{\mathrm{j}, v} \frac{\partial \Omega_{j}\left(f_{\mathrm{j}, v}\right)}{\partial f_{i, v}}
$$

\subsection{Adjoint Boundary Condition}

The adjoint boundary condition is obtained from terms of integration of Eq. (15) on the boundary $c$. Since boundary condition is singular after using conservation relations, after study, a new relation between the pressure and distribution function for inviscid adjoint boundary condition is presented as follow:

$$
p=\sum_{i=1}^{M} \sum_{v=1}^{L}\left(\frac{f_{i, \nu}\left(a e_{i 1}+b e_{i 2}\right)\left(e_{i 1}+e_{i 2}\right)}{a+b}\right)
$$

and

$$
\begin{aligned}
\delta p & =\sum_{i=1}^{M} \sum_{v=1}^{L}\left(\frac{\left(a e_{i 1}+b e_{i 2}\right)\left(e_{i 1}+e_{i 2}\right)}{a+b}\right) \delta f_{i, v} \\
& +\sum_{i=1}^{M} \sum_{v=1}^{L} f_{i, v} \delta\left(\frac{\left(a e_{i 1}+b e_{i 2}\right)\left(e_{i 1}+e_{i 2}\right)}{a+b}\right)
\end{aligned}
$$

where $a=\frac{\partial \eta}{\partial x}, \quad b=\frac{\partial \eta}{\partial y}$

Value of the $\psi_{i, v}$ on the boundary $c$ can be derived by making independent of Eq. (15) from flow variation:

$$
\begin{aligned}
\psi_{i, v}=-\frac{\left(e_{i 1}+e_{i 2}\right)}{q_{\infty} J(a+b)}[ & \Phi\left(S_{22} \cos \alpha+S_{21} \sin \alpha\right) \\
& \left.-\left(S_{22} \sin \alpha-S_{21} \cos \alpha\right)\right]
\end{aligned}
$$

Finally, by substituting the Eq. (19) in Eq. (15), the final variation of the objective function can be simplified as:

$$
\begin{aligned}
\delta I= & -\frac{1}{q_{\infty} \bar{c}} \sum_{i=1}^{M} \sum_{v=1}^{L} \oint_{c} f_{i, v} \delta\left(\frac{e_{i 2}^{2}}{a^{2}+b^{2}}\right) \\
& {\left[\Phi\left(S_{22} \cos \alpha+S_{21} \sin \alpha\right)\right.} \\
& \left.-\left(S_{22} \sin \alpha-S_{21} \cos \alpha\right)\right] d \xi \\
& -\oint_{c} C_{p}\left[\Phi\left(\delta S_{22} \cos \alpha+\delta S_{21} \sin \alpha\right)\right. \\
& \left.+\left(\delta S_{22} \sin \alpha-\delta S_{21} \cos \alpha\right)\right] d \xi \\
& +\sum_{i=1}^{M} \sum_{\nu=1}^{L} \int_{D} \psi_{i, v}\left[\frac{\partial f_{i, v}}{\partial \xi} \delta\left(J e_{i 1}\right)+\right. \\
& \left.\frac{\partial \delta f_{i, v}}{\partial \eta} \delta\left(J e_{i 2}\right)-\Omega_{i, v}\left(f_{\mathrm{i}, \nu}\right) \delta J\right] d \xi d \eta
\end{aligned}
$$

\section{EQUATIONS DISCRETIZATION}

\subsection{Flow Equation}

To discretize the lattice Boltzmann equation (LBE) for flow simulation using the cell centered finite volume method, the conservative form should be used [Qu et al. (2007)]:

$\frac{\partial f_{i, v}}{\partial t}+\frac{\partial F_{i, v}}{\partial \xi}+\frac{\partial G_{i, v}}{\partial \eta}=\Omega_{\alpha, v}$

$\Omega_{\alpha, v}=\frac{f_{i, v}^{e q}-f_{i, v}}{\tau}$

where $\Omega_{\alpha, v}$ is the collision operation and $F$ and $\mathrm{G}$ are the convection fluxes in $\xi$ and $\eta$ directions, respectively. The finite volume discrete form of the lattice Boltzmann equation can be written as:

$f_{i, j}^{n+1}=f_{i, j}^{n}-\frac{\Delta t}{V o l_{i, j}}\left\{\left(F_{i+\frac{1}{2}, j}^{n} \Delta s_{i+\frac{1}{2}, j}+\right.\right.$ $\left.\left.F_{i-\frac{1}{2}, j}^{n} \Delta s_{i-\frac{1}{2}, j}\right)\left(G_{i, j+\frac{1}{2}}^{n} \Delta s_{i, j+\frac{1}{2}}+G_{i, j-\frac{1}{2}}^{n} \Delta s_{i, j-\frac{1}{2}}\right)\right\}+$ $\Delta t \Omega_{i, j}$

In Eq.

$i, j$ are the cell centers, $V o l_{i, j}$ represents the cell volume, $F_{i \pm \frac{1}{2}, j}$ and $G_{i, j \pm \frac{1}{2}}$ are the numerical fluxes and $\Delta s$ is the cell surface.

Numerical fluxes can be calculated by the Riemann solver and interpolation methods. To capture the shock waves in numerical simulations of the compressible flow, the numerical dissipation or artificial viscosity is applied to the solver.

For spatial discretization of the numerical fluxes, the $3^{\text {rd }}$ order of MUSCL method is used with a smooth limiter to interpolate the fluxes on both sides of the cell surface. For example, $F$ fluxe can be discretized 
as:

$$
F_{i+\frac{1}{2}, j}^{n}=\left\{\begin{array}{c}
\left(f_{L}\right)_{i+\frac{1}{2}, j}\left(\boldsymbol{e}_{\alpha \xi} \cdot \boldsymbol{N}_{i+\frac{1}{2}, j}\right) \\
i f\left(\boldsymbol{e}_{\alpha \xi} \cdot \boldsymbol{N}_{i+1 / 2, j}\right) \geq 0 \\
\left(f_{R}\right)_{i+\frac{1}{2, j}}\left(\boldsymbol{e}_{\alpha \xi} \cdot \boldsymbol{N}_{i+\frac{1}{2}, j}\right) \\
i f\left(\boldsymbol{e}_{\alpha \xi} \cdot \boldsymbol{N}_{i+1 / 2, j}\right) \leq 0
\end{array}\right.
$$

where $\boldsymbol{N}_{i+1 / 2, j}$ is the surface normal vector. The left $\left(f_{L}\right)$ and right $\left(f_{R}\right)$ distribution functions of the surface can be written as:

$$
\left\{\begin{array}{c}
\left(f_{L}\right)_{i+\frac{1}{2}, j}= \\
f_{i, j}+\left\{\frac{s}{4}\left[(1-k s) \Delta_{-}+(1+k s) \Delta_{+}\right]\right\}_{i} \\
\left(f_{R}\right)_{i+\frac{1}{2}, j}= \\
f_{i+1, j}-\left\{\frac{s}{4}\left[(1-k s) \Delta_{+}+(1+k s) \Delta_{-}\right]\right\}_{i+1}
\end{array}\right.
$$

where $k$ determines accuracy of the interpolation. For example, $k=1 / 3,0,1$ gives $3^{\text {rd }}$, upwind $2^{\text {nd }}$ and central $2^{\text {nd }}$ order of interpolations, respectively. The Van Albada limiter, $s$, is also defined as:

$S=\frac{2 \Delta_{+} \Delta_{+}+\varepsilon^{2}}{\Delta_{+}^{2}+\Delta_{-}^{2}+\varepsilon}$

where $\varepsilon$ has a very small value about $10^{-6}$ and the $\Delta$ operator is expressed as:

$\left(\Delta_{+}\right)_{i}=f_{i+1, j}-f_{i, j}\left(\Delta_{-}\right)_{i}=f_{i, j}-f_{i-1, j}$

Other numerical fluxes are also calculated in the same way.

\subsection{Adjoint Equation}

In order to discretize the adjoint lattice Boltzmann equation (ALBE) for calculation of the objective function gradients which are independent to number of the design variables, the same method applied to the LBE is used. Therefore, the ALBE in conservative form in $2 \mathrm{D}$ can be written as:

$\frac{\partial \psi_{i, v}}{\partial t}+\frac{\partial \mathcal{F}_{i, v}}{\partial \xi}+\frac{\partial \mathcal{R}_{i, v}}{\partial \eta}=\frac{1}{\tau}\left(\psi_{i, v}-\psi_{i, v}^{e q}\right)$

where $\mathcal{F}$ and $\mathcal{R}$ are the adjoint convection fluxes in $\xi$ and $\eta$ directions, respectively. The finite volume discrete form of the adjoint lattice Boltzmann equation can be written as:

$$
\begin{aligned}
& \psi_{i, j}^{n-1}=\psi_{i, j}^{n}+\frac{\Delta t}{\operatorname{Vol}_{i, j}}\left\{\left(\mathcal{F}_{i+\frac{1}{2}, j}^{n} \Delta s_{i+\frac{1}{2}, j}+\right.\right. \\
& \left.\left.\mathcal{F}_{i-\frac{1}{2}, j}^{n} \Delta s_{i-\frac{1}{2}, j}\right)\left(\mathcal{R}_{i, j+\frac{1}{2}}^{n} \Delta s_{i, j+\frac{1}{2}}+\mathcal{R}_{i, j-\frac{1}{2}}^{n} \Delta s_{i, j-\frac{1}{2}}\right)\right\}- \\
& \Delta t\left(\frac{1}{\tau}\left(\psi_{i, j}-\psi_{i, j}^{e q}\right)\right)
\end{aligned}
$$

Also, after discretization, the same numerical techniques can be applied to solve Eq. (32).

At the microscopic level, one can solve the FV-LBM to obtain solution of $f_{i, v}$. One then takes the moments to obtain the microscopic variables at any times $0 \leq$ $t \leq T_{\text {steady }}$. These are then used to solve the mesoscopic adjoint equation backward in time for the adjoint variable $\psi_{i, v}$. The solution is used together with optimal condition to obtain the gradient of the objective function respect to the design variables.

\section{OPTIMIZATION ALGHORITM}

The optimization procedure can be presented as following steps:

step 1. Solve the flow field governing equation, Eq. (26), and calculate $f_{i, v}$, forward in time for $\alpha$ and $\alpha+\Delta \alpha$.

step 2. Calculate $C_{l_{\alpha}}$ and $C_{d_{\alpha}}$.

step 3. Calculate constant $\Phi$ from Eq. (11).

step 4. Solve the adjoint equation, Eq. (32), backward in time to compute $\psi_{i, v}$ for both the objective functions.

step 5. Calculate the gradient vector of main objective function using Eq. (23).

step 6. Modify airfoil shape using steepest descent method, $y_{i, 1}^{\text {new }}=y_{i, 1}^{\text {old }}-\lambda \times \nabla \mathrm{I}$ (where $\lambda$ is optimization step size).

step 7. Calculate the gradient vector of $I^{\prime}$ objective function.

step 8. Modify the angle of attack using Eq. (9).

step 9. Modify the grid using Jamson's method.

step 10. Return to Step 1 until the optimization convergence criterion is satisfied.

For clarification, the flowchart of the present algorithms of airfoil optimization is shown in Fig. 1 .

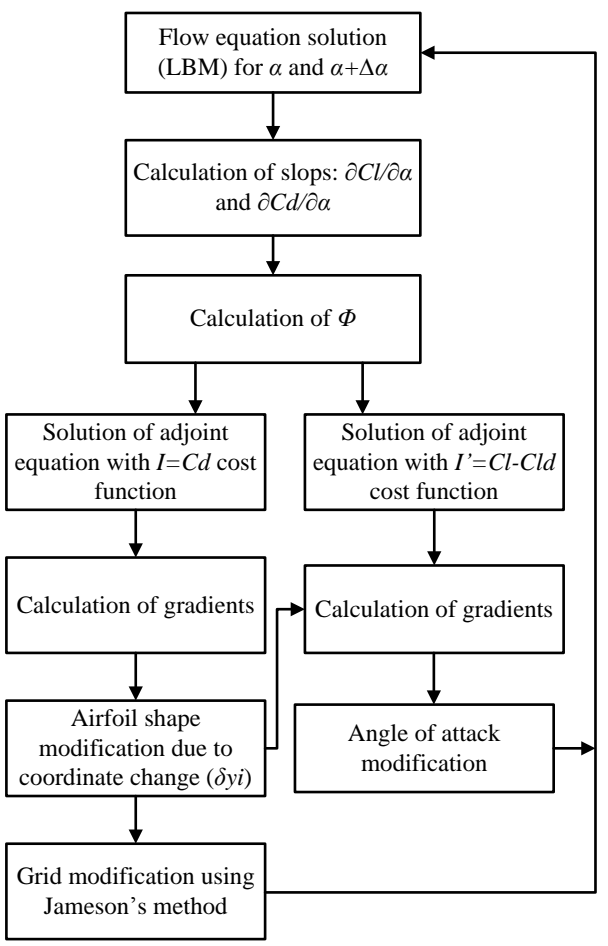

Fig. 1. Flowchart of optimization algorithm for airfoil optimization. 


\section{NUMERICAL RESULTS}

To determine capability of the derived AFV-LBM and optimization algorithm, the supercritical $\mathrm{SC}(2) 0410$ airfoil is numerically optimized at cruise conditions based on minimizing the drag coefficient by eliminating the wave drag with constraint of fixed lift coefficient.

\subsection{Airfoil Optimization}

In this section, the supercritical airfoil SC(2)-0410 is optimized using the developed algorithm at $M_{\infty}=0.758$ and $\alpha=1.29$ in inviscid conditions.

The cost function is to minimize the drag coefficient and the optimization constraint is to keep fixed the lift coefficient at $C_{l}=0.641$. The design variables in this problem are the angle of attack $(\alpha)$ and $\mathrm{y}$ coordinates of the airfoil surface. Convergence history of the drag coefficient reduction, lift coefficient constraint and angle of attacks during 20 design cycle has been presented in Figs. 2 to 4 . The results indicate that the optimization goal in minimizing the drag coefficient and keeping fixed the lift coefficient are satisfied after 20 design cycles. Geometric profiles of the base and optimized airfoil after 20 design cycles have been compared in Fig. 5.

The optimal airfoil design has been performed using the developed optimization algorithm in inviscid conditions. Comparison of the Mach number contours for both the base and optimized airfoil in Fig. 6 indicates that the strong shock wave is eliminated in new airfoil profile. Some oscillations in contour lines are due to y coordinate movement of surface points during the optimization. Comparison of the pressure distributions on the base and optimal airfoil in Fig. 7 also confirms that the optimization approach can eliminate the upper shock wave on the airfoil surface.

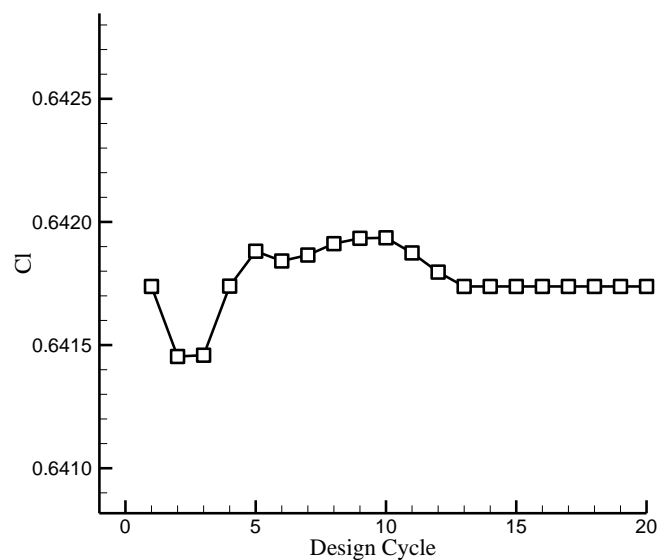

Fig. 2. Lift coefficient history during 20 design cycles.

To show accuracy of the developed optimization algorithm to achieve our goals in wave drag reduction, the coefficients values have been presented in table 1. It is obvious that the lift coefficient has remained constant for the optimized airfoil profile and the drag coefficient has been reduced about $49 \%$ by neglecting the wave drag. Totally, this optimization improves the airfoil performance in $\mathrm{Cl} / \mathrm{Cd}$ about $106 \%$. It is noted that the angle of attack has been also decreased to 1 degree during the optimization process because it is selected as a design variable.

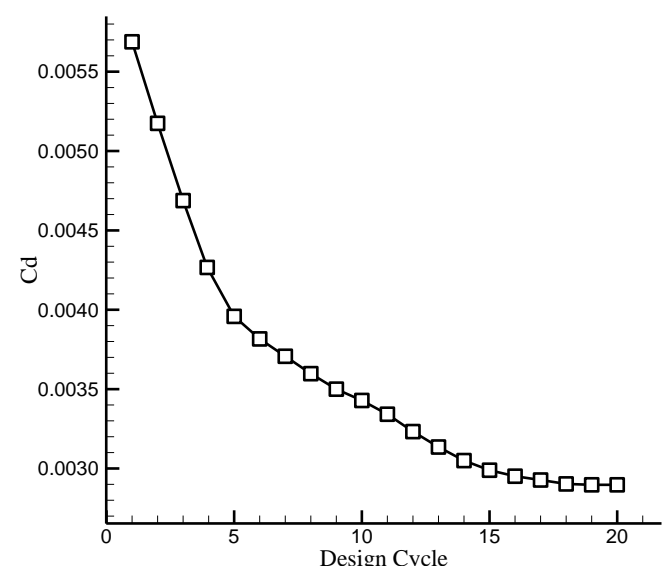

Fig. 3. Drag coefficient reduction history during 20 design cycles.

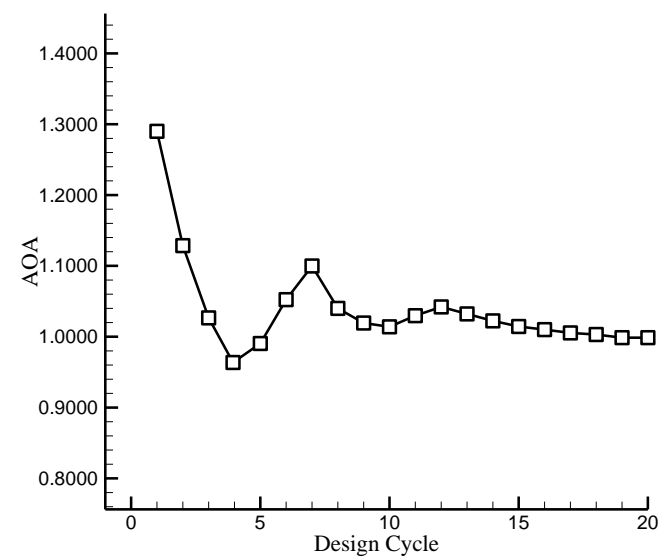

Fig. 4. Angle of attack history during 20 design cycles.

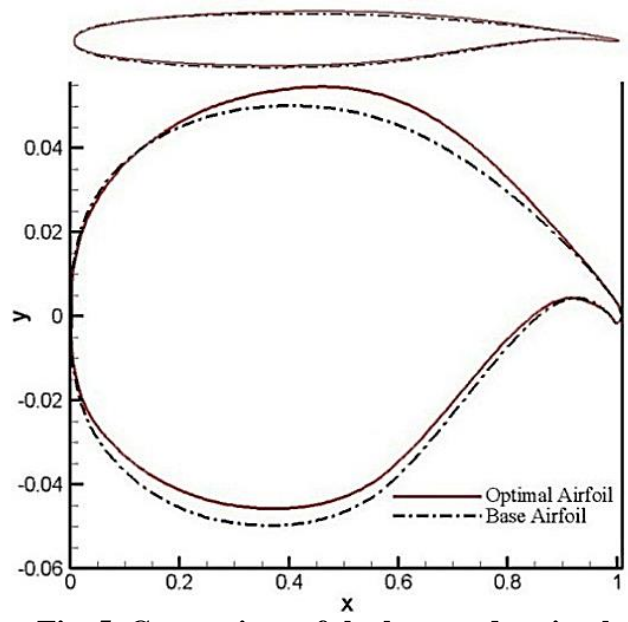

Fig. 5. Comparison of the base and optimal airfoil profile. 


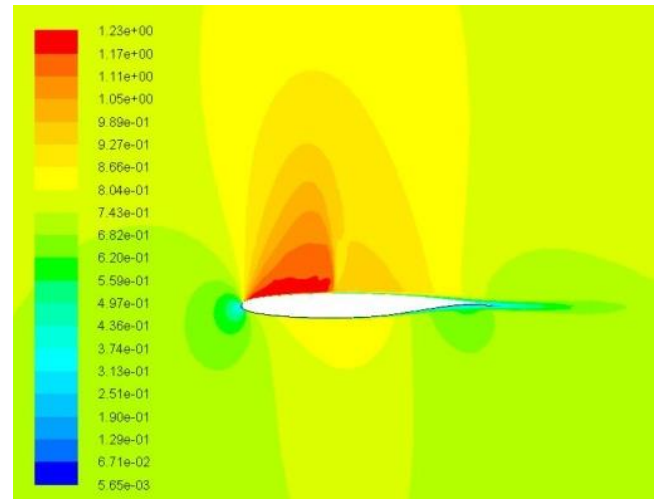

(a)

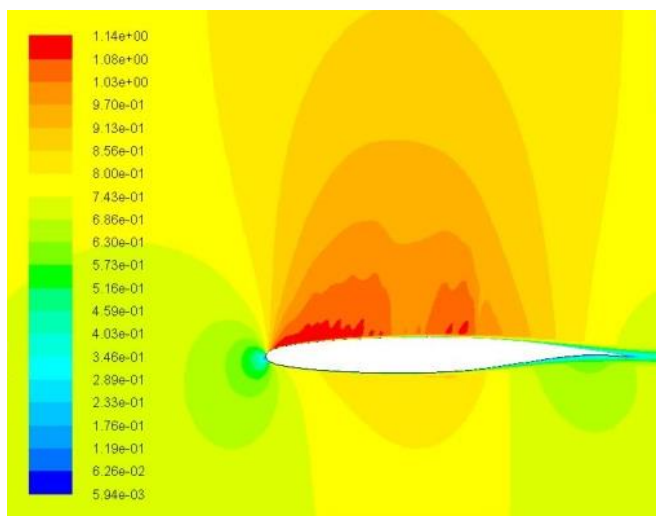

(b)

Fig. 6. Mach number contours around the base and optimized airfoil. (a) Base airfoil (b) Optimal airfoil.

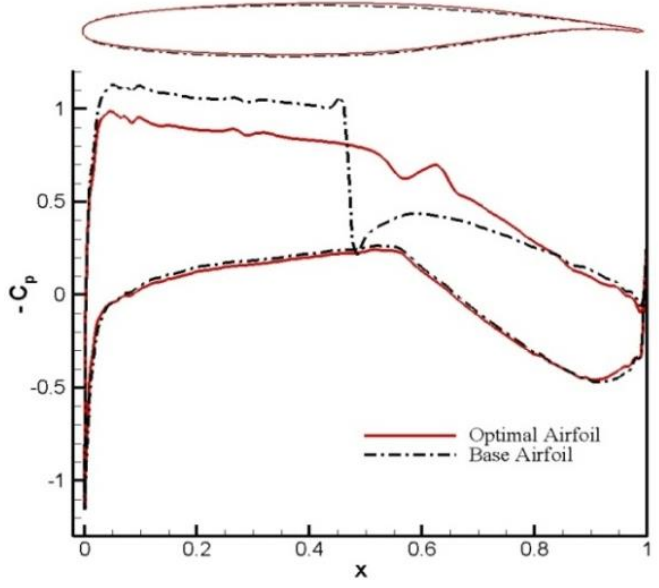

Fig. 7. Comparison of the pressure distribution on the base and optimal airfoil.

Table 1 Comparison of the base and optimal aerodynamic coefficients at design conditions

\begin{tabular}{|c|c|c|c|}
\hline Parameter & $\begin{array}{c}\text { Base } \\
\text { Airfoil }\end{array}$ & $\begin{array}{c}\text { Optimal } \\
\text { Airfoil }\end{array}$ & $\begin{array}{c}\text { Percent of } \\
\text { Optimization }\end{array}$ \\
\hline CL & 0.641 & 0.641 & Constant \\
\hline CD & 0.00568 & 0.00289 & $49 \%$ \\
\hline CL/CD & 112.82 & 232.94 & $106 \%$ \\
\hline AOA & 1.29 & 0.998 & \\
\hline
\end{tabular}

\section{WIND TUNNEL TEST}

Since the optimization is numerically performed at inviscid conditions, the experimental test has been defined to find effect of viscosity in obtained results. The results indicate that the viscosity makes the shock position on the airfoil surface closer to the nose and causes the shock become weaker than that in inviscid condition. Thus, it is expected that optimization performance designed at inviscid conditions does not have the same efficiency. The main goal of the experimental test is to find variation of optimization performance due to real condition.

\subsection{Experimental Set Up}

The experimental test has been performed in a high speed suction wind tunnel with a test chamber of $0.6 \times 0.6 \mathrm{~m}^{2}$ with a Mach number of 0.4 to 0.85 (see Fig. 8). The free steam turbulence level is $0.5 \%$ at the most. The chamber's walls are porous which are adjustable up to $6 \%$. The tests were performed under the conditions and specifications presented in table 2.

Table 2 Flow Conditions

\begin{tabular}{|c|c|}
\hline Parameters & Value \\
\hline Mach number & 0.76 \\
\hline Angle of Attack (Base airfoil) (Deg.) & 1.2 \\
\hline $\begin{array}{c}\text { Angle of Attack (Optimal airfoil) } \\
\text { (Deg.) }\end{array}$ & 1 \\
\hline Free stream pressure (Pa) & 57600 \\
\hline Free stream dynamic pressure (Pa) & 23062 \\
\hline Free stream temperature (K) & 300 \\
\hline
\end{tabular}

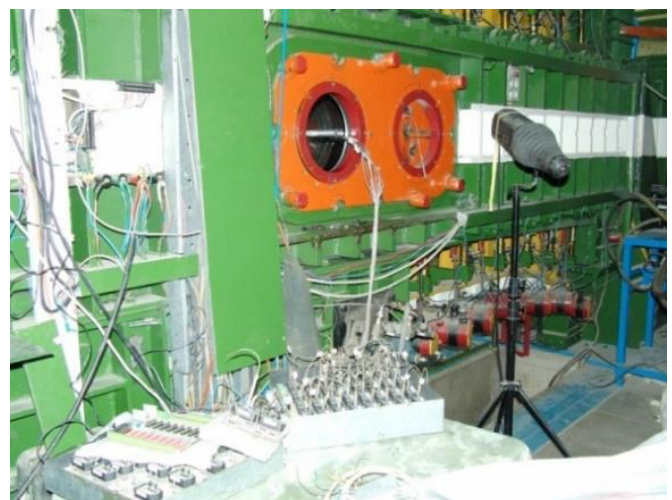

Fig. 8. Transonic wind tunnel and experimental equipment.

The test plan has been involved measuring the pressure distribution on the airfoil upper and lower surfaces, and qualitative analysis of output data. The Mach number measurements, during the tests, were done with a pitot-static tube fixed at the model upstream whit maximum calculation error of $1 \%$. The pressures were measured using differential pressure sensors with a measurement range of up to 15 psi and maximum error of $0.15 \%$ span. The pressure sensors uncertainty from the sum of the random error and the systematic error, in measuring the pressure and calculating the pressure coefficient was $2.1 \%$. 
The base model used is a NASA-SC-0410 supercritical airfoil with a chord length of $20 \mathrm{~cm}$, thickness of $2 \mathrm{~cm}$, and span of $60 \mathrm{~cm}$, made of VCN steel (see Fig. 9). To measure the pressure distribution on the upper and lower surfaces, 32 pressure taps for optimal airfoil and 40 pressure taps for base airfoil have been used, congested more at the leading edge.

The tests error sources include the flow field (both non-uniformity and turbulence level in the wind tunnel), model installation and $\alpha$-setting, pressure sensors, airfoil model making, linear potentiometer, $\mathrm{A} / \mathrm{D}$ range, and pressure points locations on the airfoil, effects of walls, and model blockage. The maximum uncertainty of $4 \%$ was calculated and applied to the obtained results (Haghiri et al. 2015).
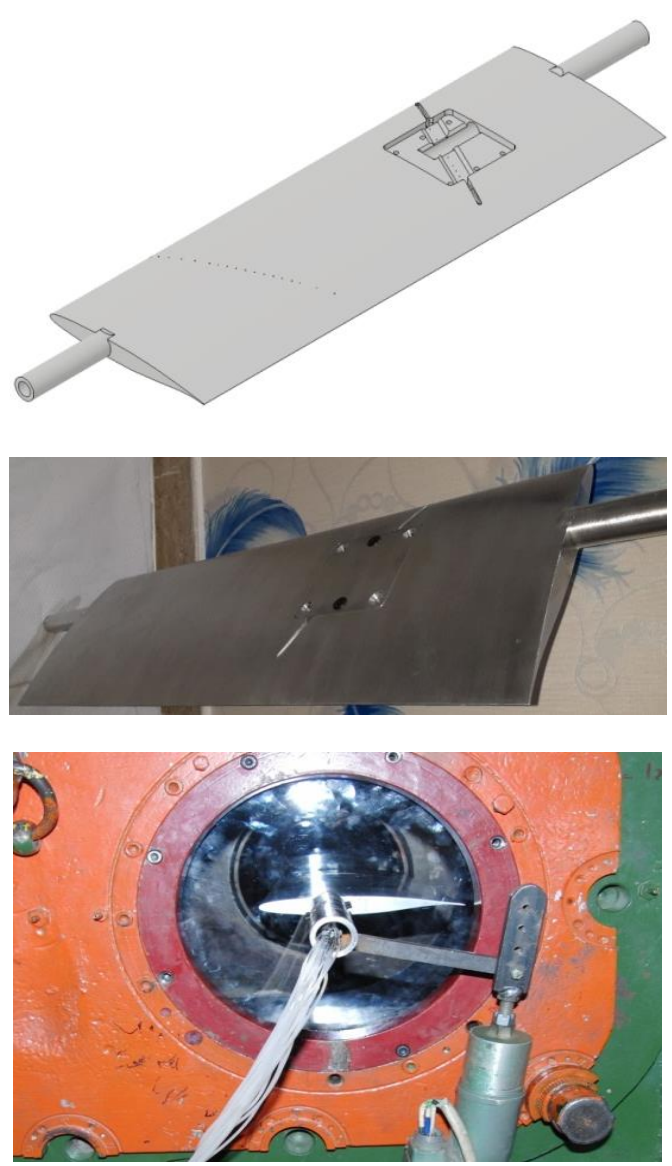

Fig. 9. Optimal airfoil model fixed in the tunnel.

\subsection{Experimental Results}

To find effect of viscosity and turbulent flow in obtained optimal supercritical airfoil, the numerical simulation and experimental test have been performed at real conditions in the transonic wind tunnel.

Firstly, the base airfoil (SC(2)-0410) has been experimentally tested at transonic cruise conditions, $\mathrm{M}=0.76$ and angle of attack of 1.2 degree. Figure 10 shows comparison of numerical simulation and experimental results. The Mach number contours prepared from numerical results and shadowgraph from experimental test indicates that there is a strong shock on the top surface of base airfoil. The pressure distributions on the airfoil shows the shock occurs at about $\mathrm{X} / \mathrm{C}=0.3$. Good agreement of the experimental and numerical results demonstrates correct trend of this study.

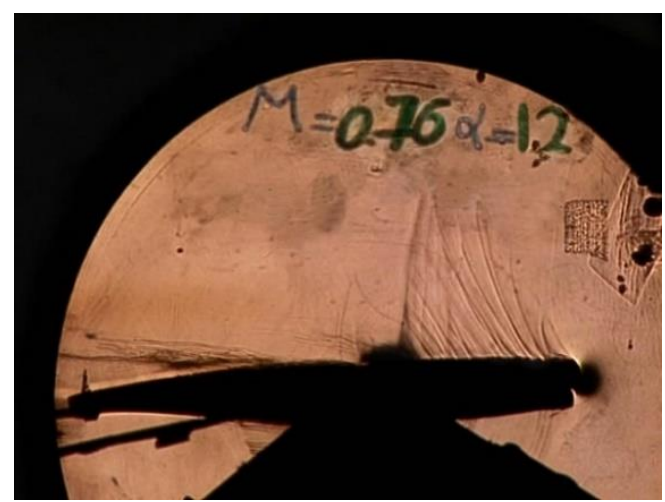

(a)

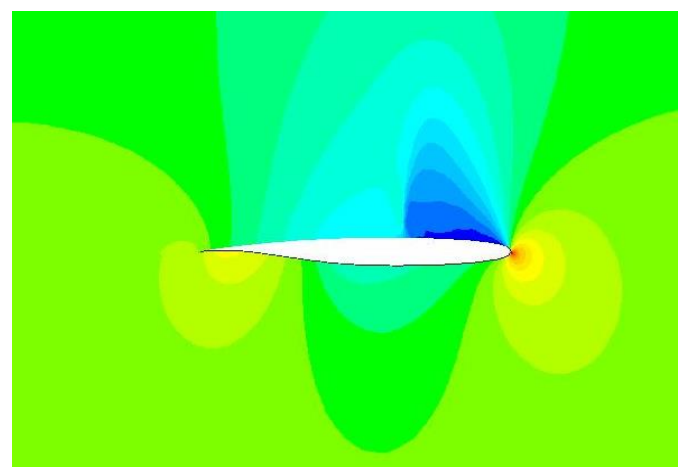

(b)

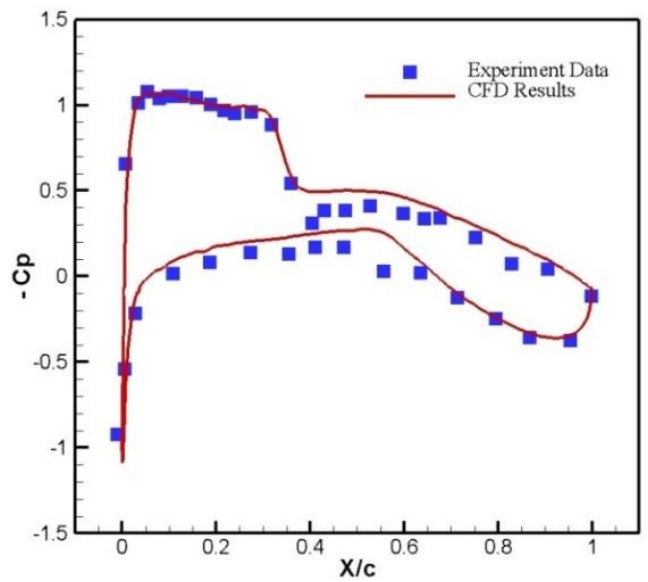

(c)

Fig. 10. Experimental and numerical results around the base airfoil (a) Shadowgraph visualization, (b) Pressure Contours, (c) Comparison of the pressure distributions.

Secondly, the optimal airfoil, obtained from inviscid optimization algorithm, has been experimentally tested at transonic optimal conditions, $\mathrm{M}=0.76$ and angle of attack of 1 degree. Again, the Mach number contours from numerical simulation and experimental shadowgraph and comparison of pressure distributions on the airfoil surface have been shown in Fig. 11. The good agreement of the 
numerical and experimental results is also seen in this test. Some important points can be understood from these results. Firstly, as obtained from inviscid optimal airfoil design, the shock is also eliminated from physical domain at real conditions in the wind tunnel. The extracted pressure distribution from the experimental test is well shown this fact. Secondly, the surface point's movement in y coordinate during the optimization process creates a non-smoothed surface which causes some Mach waves and oscillations on top of airfoils. Thirdly, gathering these Mach waves on top surface of optimal airfoil make an expansion wave where located at about $\mathrm{X} / \mathrm{C}=0.6$.

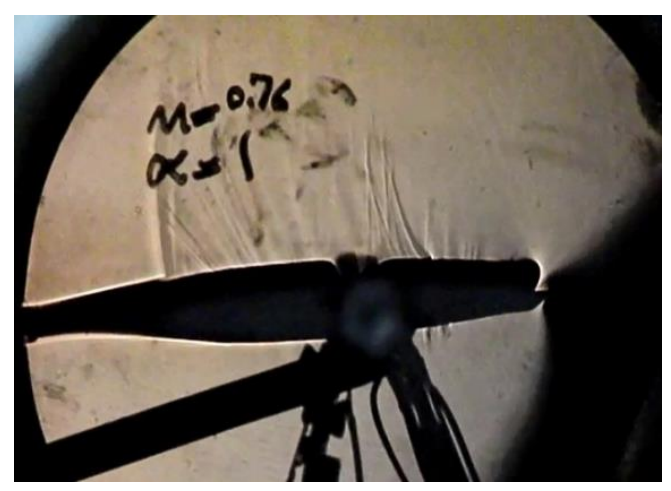

(a)

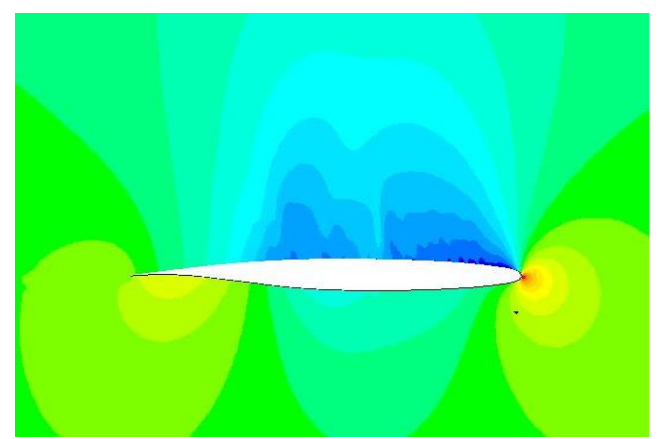

(b)

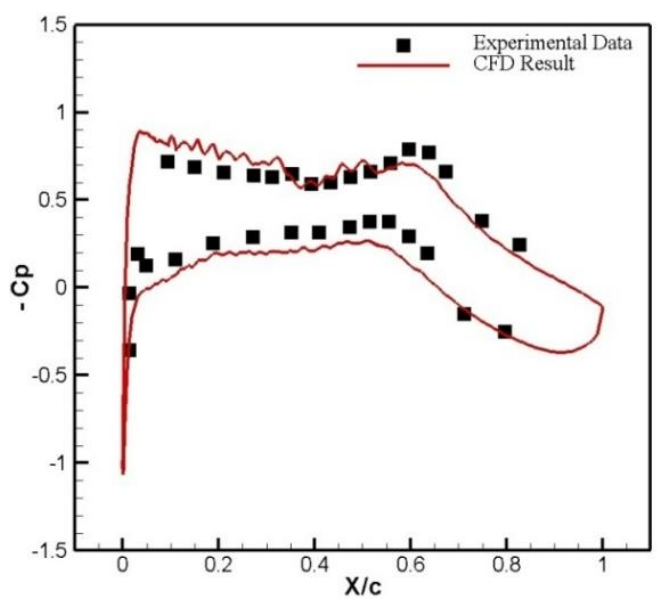

(c)

Fig. 11. Experimental and numerical results around the optimal airfoil (a) Shadowgraph visualization, (b) Pressure Contours, (c) Comparison of the pressure distributions.
To ensure the accuracy of numerical simulation in presented results, the grid study is performed using comparison of the pressure distribution for three types of grid including coarse, medium and fine meshes for both the original and optimal models (Fig. 12). The total elements of coarse, medium and fine grids are about 30,000, 70,000 and 240,000, respectively. To accurately capture wall effects, the boundary layer grid and standard wall function have been applied near the wall for numerical simulation. The first boundary layer row has $0.02 \mathrm{~mm}$ distance from the wall and 20 rows of the boundary layer mesh with 1.1 aspect ratio create maximum $\mathrm{Y}+$ about 15 near the wall.

The captured Y+ makes us sure that the turbulent structure are well captured. The results show that the medium grid is proper to numerical simulation. All of results presented in comparison with experimental data have been extracted by the medium grid.

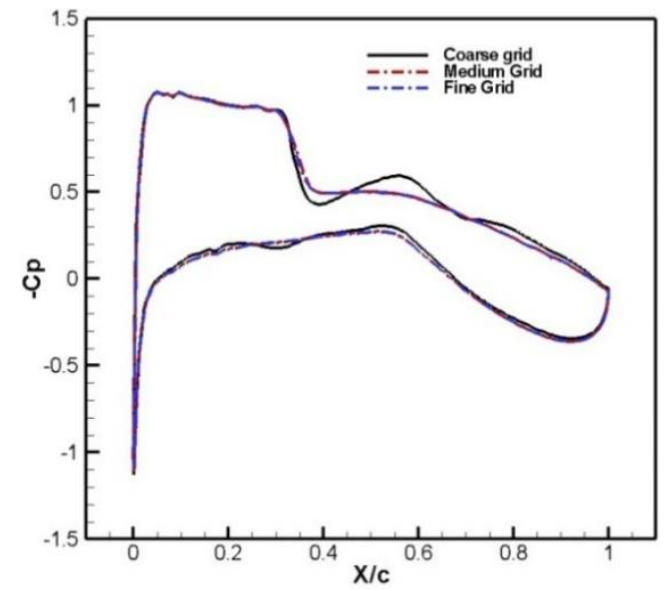

(a) Base Model

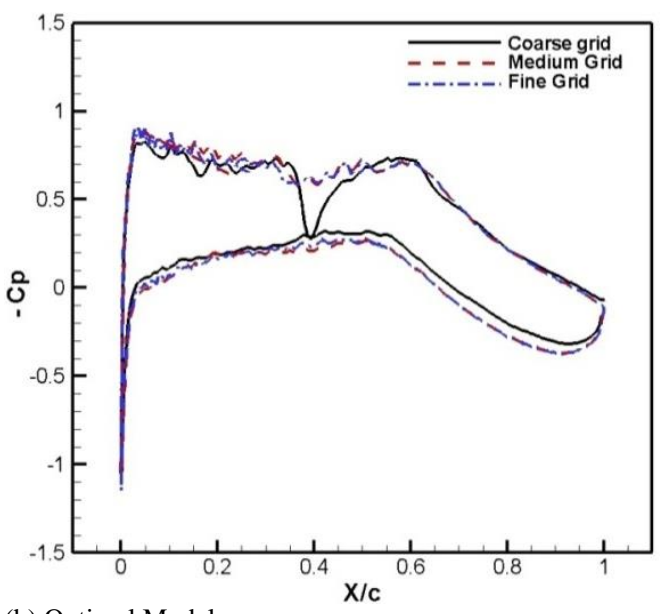

(b) Optimal Model

Fig. 12. Grid study of surface pressure distribution for numerical simulation.

To more accurate comparison, summary of obtained results at real conditions have been presented in table 3. It is found that the designed optimal airfoil has better performance rather than the base airfoil even at real conditions, where the lift coefficient has been kept fixed and the drag coefficient has been reduced about $22 \%$ due to eliminating the shock. Although 
R. Kamali Moghadam et al. / JAFM, Vol. 13, No. 4, pp. 1277-1287, 2020.

Table 3 Comparison of the base and optimal aerodynamic coefficients at real conditions

\begin{tabular}{|c|c|c|c|}
\hline Parameter & Based Airfoil & Optimized Airfoil & Percent of Optimization \\
\hline CL & 0.532 & 0.5296 & $-0.4 \%$ \\
\hline CD & 0.0134 & 0.01049 & $22 \%$ \\
\hline CL/CD & 39.61 & 50.46 & $27 \%$ \\
\hline AOA & 1.2 & 1 & \\
\hline
\end{tabular}

the designed optimal airfoil has supplied our goals in drag reductions, it has lower performance at real conditions rather than the inviscid design conditions. It is indicated that the viscosity and turbulence conditions has reduced the designed airfoil performance about $27 \%$.

\section{CONCLUSION}

The present research includes two main parts. The first part is to develop a new adjoint compressible lattice Boltzmann method to geometrically optimize a supercritical airfoil in inviscid conditions. The second part is to experimentally investigate the obtained optimal airfoil profile in the transonic wind tunnel in real conditions. Minimizing the drag coefficient and eliminating the shock wave on the supercritical airfoil surface are considered as the cost function with constraint of fixed lift coefficient.

In developed code, the circular function is chosen as distribution function instead of the Maxwell distribution function due to its mathematical simplicity and flexibility of new lattice definition in simulation of compressible flowfield. The continuous adjoint method is applied for calculation of objective function gradient vector. Also, the finite volume method is utilized to discretize the governing equation which is suitable for optimization of both the compressible and incompressible inviscid flow. The supercritical SC(2)0410 airfoil has been optimized using the developed code at inviscid condition.

For validation of the developed algorithm, the optimal airfoil has been experimentally tested in transonic wind tunnel with viscosity effect. Pressure distribution on the surface of both the base and optimized airfoil are extracted from experimental tests and compared with those of numerical simulations. Therefore, effect of viscosity is investigated in eliminating the shock wave of optimized airfoil as the cost function. As obtained from inviscid optimal airfoil design, the shock is also eliminated from physical domain at real conditions in the wind tunnel. It is also found that the designed optimal airfoil has better performance rather than the base airfoil even at real conditions, where the lift coefficient has been kept fixed and the drag coefficient has been reduced about $22 \%$.

\section{REFERENCES}

Alexander, F., H. Chen and G. D. Doolen (1992). Lattice Boltzmann model for compressible fluids, Physical Review A 46(4), 1967.

Anderson, W. K. and V. Venkatakrishnan (1999).
Aerodynamic design optimization on unstructured grids with a continius adjoint formulation, Computers and Fluids 28 (4), 443480 .

Chenghai, S. and A. T. Hsu (2003). Threedimensional lattice Boltzmann model for compressible flows, Physical Review E 68(1), 016303 .

Farrokhfal, H. and A. R. Pishevar (2014). Optimization of Airfoils for Minimum Pitching Moment and Compressibility Drag Coefficients, Journal of Aerospace Technology and Management 4, 395-406.

Frisch, U., D. Humieres, P. Lallemand, B. Hasslacher and Y. Pomeau (1987). Lattice Gas Hydrodynamics in Two and Three Dimensions, Complex Systems 1, 649-707.

Haghiri, A. A., M. Mani and N. Fallahpour (2015). Unsteady boundary layer measurement on an oscillating (pitching) supercritical airfoil in compressible flow using multiple hot-film sensors, Proceedings of the Institution of Mechanical Engineers, Part G: Journal of Aerospace Engineering 229(10), 1771-1784.

Hardy, J., Y. Pomeau and O. DePazzis (1973). Time evolution of a two-dimensional model system. I. Invariant states and time correlation functions, Journal of Mathematical Physics 14(12), 17461759.

He, Y., Q. Liu and Q. Li (2013). Three-dimensional finite-difference lattice Boltzmann model and its application to inviscid compressible flows with shock waves. Physica A: Statistical Mechanics and its Applications 392(20), 4884-4896.

Jameson, A. (1988). Aerodynamic design via control theory. Journal of Scientific Computing 3(3), 233-260.

Jameson, A. and K. Leoviriyakit (2003). Aerodynamic Shape optimization of wing including planform variations, AIAA paper, Aerospace Science Meeting and Exhibit, Reno, Nevada.

Jameson, A. and K. Leoviriyakit (2005). Multi-point wing planform optimization via control theory, in $43^{r d}$ Aerospace Sciences Meeting and Exhibit, Reno, Nevada.

Kadanoff, L., P. Leo, G. R. McNamara and G. Zanetti (1989). From automata to fluid flow: Comparisons of simulation and theory, Physical Review A 40(8), 4527.

Kreissl, S., Pingen, G. and Maute, K. (2011). An 
R. Kamali Moghadam et al. / JAFM, Vol. 13, No. 4, pp. 1277-1287, 2020.

explicit level set approach for generalized shape optimization of fluids with the lattice Boltzmann method. International Journal for Numerical Methods in Fluids 65(5), 496-519.

Kun, X. (2001). A gas-kinetic BGK scheme for the Navier-Stokes equations and its connection with artificial dissipation and Godunov method, Journal of Computational Physics 171(1), 289335.

Leoviriyakit, K. (2005), Wing Optimization via an Adjoint Method, $\mathrm{PhD}$ Thesis, Stanford University.

Li, X., L. Fang and Y. Peng (2018). Airfoil design optimization based on lattice Boltzmann method and adjoint approach, Applied Mathematics and Mechanics 39(6), 891-904.

Meng, J., N. Dongari, J. M. Reese and Y. Zhang (2014). Breakdown parameter for kinetic modeling of multi-scale gas flows, Physical Review E, 89, 063305.

Hekmat, M. H. and M. Mirzaei (2015). Extraction of macroscopic and microscopic adjoint concepts using a lattice Boltzmann method and discrete adjoint approach, Physical Review E 91(1), 013303.

Hekmat, M. H. and M. Mirzaei (2015) A comparison of the continuous and discrete adjoint approach extended based on the standard lattice Boltzmann method in flow field inverse optimization problems, Acta Mechanica 227(4), 015-1509.

Nadarajah, S. K., A. Jameson and M. S. McMullen, (2003). Optimum shape design for unsteady flows using time accurate and non-linear frequency domain methods. (Vol. 3875). AIAA.

Ngnotchouye, J. M. T., M. Herty, S. Steffensen and M. K. Banda (2011), Relaxation approaches to the optimal control of the Euler equations, Computational and Applied Mathematics 30(2), 399-425.

Pingen, G., A. Evgrafov and K. Maute (2009). Adjoint parameter sensitivity analysis for the hydrodynamic lattice Boltzmann method with applications to design optimization, Computers
\& Fluids 38(4), 910-923.

Pingen, G., Evgrafov, A. and Maute, K. (2008). A parallel Schur complement solver for the solution of the adjoint steady-state lattice Boltzmann equations: application to design optimisation. International Journal of Computational Fluid Dynamics 22(7), 457-464.

Pishevar, A. R. and H. Farrokhfal (2013). Aerodynamic shape optimization of hovering rotor blades using a coupled free wake-CFD and adjoint method, Aerospace Science and Technology 28, 21-30.

Qu, K., C. Shu and Y. T. Chew (2005). Numerical simulation of flows past a rotational circular cylinder by Taylor-series-expansion and least squares-based lattice Boltzmann method, International Journal of Modern Physics C. 16(11), 1753-1770.

Qu, K., C. Shu and Y. T. Chew (2010). Lattice Boltzmann and finite volume simulation of inviscid compressible flows with curved boundary. Advances in Applied Mathematics and Mechanics 2(5), 573-586.

Qu, K., C. Shu, and Y. T. Chew (2007). Alternative method to construct equilibrium distribution functions in lattice-Boltzmann method simulation of inviscid compressible flows at high Mach number. Physical Review E 75(3), 036706.

Reuther, J., J. J. Alonso, M. J. Rimlinger and A. Jameson (1999). Aerodynamic shape optimization of supersonic aircraft configurations via an adjoint formulation on distributed memory parallel computers. Computers and Fluids 28(4-5), 675-700.

Sun, C. (1998). Lattice-Boltzmann models for high speed flows. Physical review E 58(6), 7283.

Tekitek, M. M., Bouzidi, M., Dubois, F. and Lallemand, P. (2006). Adjoint lattice Boltzmann equation for parameter identification. Computers \& fluids, 35(8-9), 805-813.

Vergnault, E. and Sagaut, P. (2014). An adjointbased lattice Boltzmann method for noise control problems. Journal of Computational Physics 276, 39-61. 